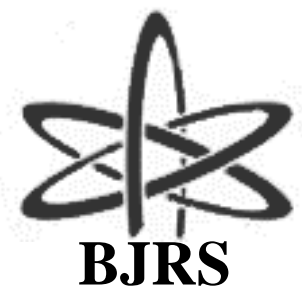

\author{
BRAZILIAN JOURNAL \\ $\mathrm{OF}$ \\ RADIATION SCIENCES \\ 08-03 (2020) 01-15
}

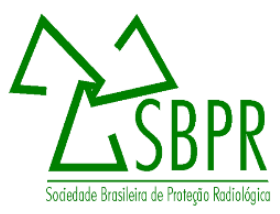

\title{
Nuclear Power Plants event classification based on International Reporting System (IRS)
}

\author{
Vital $^{\mathrm{a},{ }^{*}}$ R. B. N., Araujo ${ }^{\mathrm{a}}$ J. B., Vital ${ }^{\mathrm{b}}$ X.Y. \\ ${ }^{a}$ Comissão Nacional de Energia Nuclear (CNEN), Rua General Severiano, 90, 22290-901, Rio de Janeiro, RJ, Brasil, \\ rvital@cnen.gov.br, jeferson@cnen.gob.br \\ ${ }^{b}$ tati.mv7@gmail.com
}

\begin{abstract}
The exchange of information between several business activities can lead to an increase in productivity and quality. In nuclear area, besides economic benefits, the operating experience exchanged can increase of the safety level of nuclear installations. Thus, it's necessary to evaluate frequently operational occurrences from different countries and companies to learn with their lessons learned to prevent a recurrent events or unexpected consequences. Because this, several methodologies are recommended to investigate events in an adequate level of depth and perform reports based on local requirements. Generally, these reports contain plant conditions before and after an event occurs (operation mode, operation limits and condition, date and time, nuclear and electrical power) and their information (failure discovery methods, impact to the safety, classification from INES and national regulation scale, similar events, relation to human factors, activation of emergency plan, recommended actions, root cause and causal factors). To allow the exchange of experience the Nuclear Energy Agency (NEA) and International Atomic Energy Agency (IAEA) maintain an Incident Reporting System (IRS) that permits interaction of several member states. In this system, a local coordinator collects information of the most relevant operating events and performs an evaluation of them based on IRS Guideline presented in an annual meeting. This paper evaluates a set of approximately four hundred events reported in IRS between 2014 and 2018. This evaluation has the objective of identify the most recurrent event root causes, operation mode and the effectiveness of corrective actions in course in the nuclear industry.
\end{abstract}

Keywords: Event investigation, Operating experience, Incident Reporting System, Root cause. 


\section{INTRODUCTION}

Operational events or accidents occur in conventional industries or nuclear power plants and the impact of occurrences depends of several factors like: safety culture, training, adherence to Technical Specification, procedures, qualification of equipment, system and operators, defense in depth, redundancy and diversity [1]. Every time a failure, a transient or an accident occurs so, it's important to perform an evaluation in order to prevent recurrence of events. Event investigation has been conducted in aerospace industries as a result of big accidents [2]. In 1986, the space shuttle Challenger exploded after the launch. The investigation demonstrated that the organization decided to continue the launch even knowing a design limitation [3]. Another accident occurred in 2003 when the space shuttle Columbia returning from a mission was destroyed [3]. Several accidents were accounted in Petroleum and Gas market that caused environmental and human losses [4]. In 2005, an explosion occurred in BP Texas City Refinery resulting in fatalities, injuries and financial losses [5][6]. The investigation of this event showed deficiencies in training, safety culture, design limitations and organizational factors [6]. Another relevant accident occurred in 2010 in Deepwater Horizon, considered the largest maritime disaster in USA [7]. Considering nuclear applications, the first significant accident occurred in 1979, in Three Mile Island Nuclear Power Plants, Unit 2(TMI2), in the United States of America (USA), due the combination of equipment failure, human error and bad luck [8][9]. Another accident with big impact in nuclear community occurred in 1986 in Chernobyl Nuclear Power Plant, Unit 4, in Ukraine that investigations indicate design and technical deficiencies and, operator errors [8][10]. Recently, in 2011, a new accident occurred in Japan, in Fukushima Daiichi Nuclear Power Plant, where a combination of severe climate conditions and consequent safety system unavailability caused a release of radionuclides [8][9]. A big radiological accident occurred in 1987 in the Brazilian city of Goiania where medical equipment with radioactive source was dismantling and radionuclides were scattered contaminating several people [11].

The evaluation of the cited events gains importance when it comes specifically to nuclear power plants due to the proportions that the impact and the consequences of these events may causes to installations, workers, population and environment. Operational deviations in nuclear industry can 
promote since very little events until accidents with significant impacts. An event may be associated or influenced by many fields of knowledge, like organization (Operation, Maintenance, Engineering, Radiological Protection, Physical Protection, Chemistry, Human or Organizational Factors) or external events (earthquake, flooding, fire and other natural phenomena). This investigation needs to consider all the causes and possible consequences, to avoid the recurrence of events and improve the operational safety. Studies demonstrate that an implementation of corrective actions in an appropriate time can reduce the number of recurring events [12][13]. To improve the nuclear safety, regulatory bodies around the world define regulatory requirements related to event investigation. Brazilian regulatory body has the standard NN 1.14 that defines classes of events, types and time for notification and reporting [14]. Because of lesson learned culture, nuclear industry participates of programs to exchange operating experience. To demonstrate the importance of Event Investigation and Operating Experience Feedback System, this paper is divided in four sections. Section 2 describes methodologies to investigate events, their applications and limitations. Section 3 shows the results of events coding using IRS guideline. Section 4 presents the main conclusions and opportunities to improve the exchange of operating experience.

\section{MATERIALS AND METHODS}

In accordance with international safety practices, IAEA recommended in requirement 24 of the guide SSR-2 that: "The operating organization shall establish an operating experience programme to learn from events at the plant and events in the nuclear industry and other industries worldwide" [15]. To meet this recommendation, several methods, tools and techniques have been developed to improve investigation process. Basically, in the nuclear industry there are four event investigation methodologies as showed in Fig. 1 [16]. Root cause analysis (RCA) is a method to determine sequence of events or actions that originate an error or failure [16]. Probabilistic safety assessment enables the assessment of an installation considering potential initial events and the random behavior of component failure and human errors [17]. Deterministic safety assessment considers a sequence of events and the integration of safety system to avoid accidents based on a conservative 
input and physical model [18]. Safety culture impact assessment combines characteristic of organizations and individuals to determine the safety level [16].

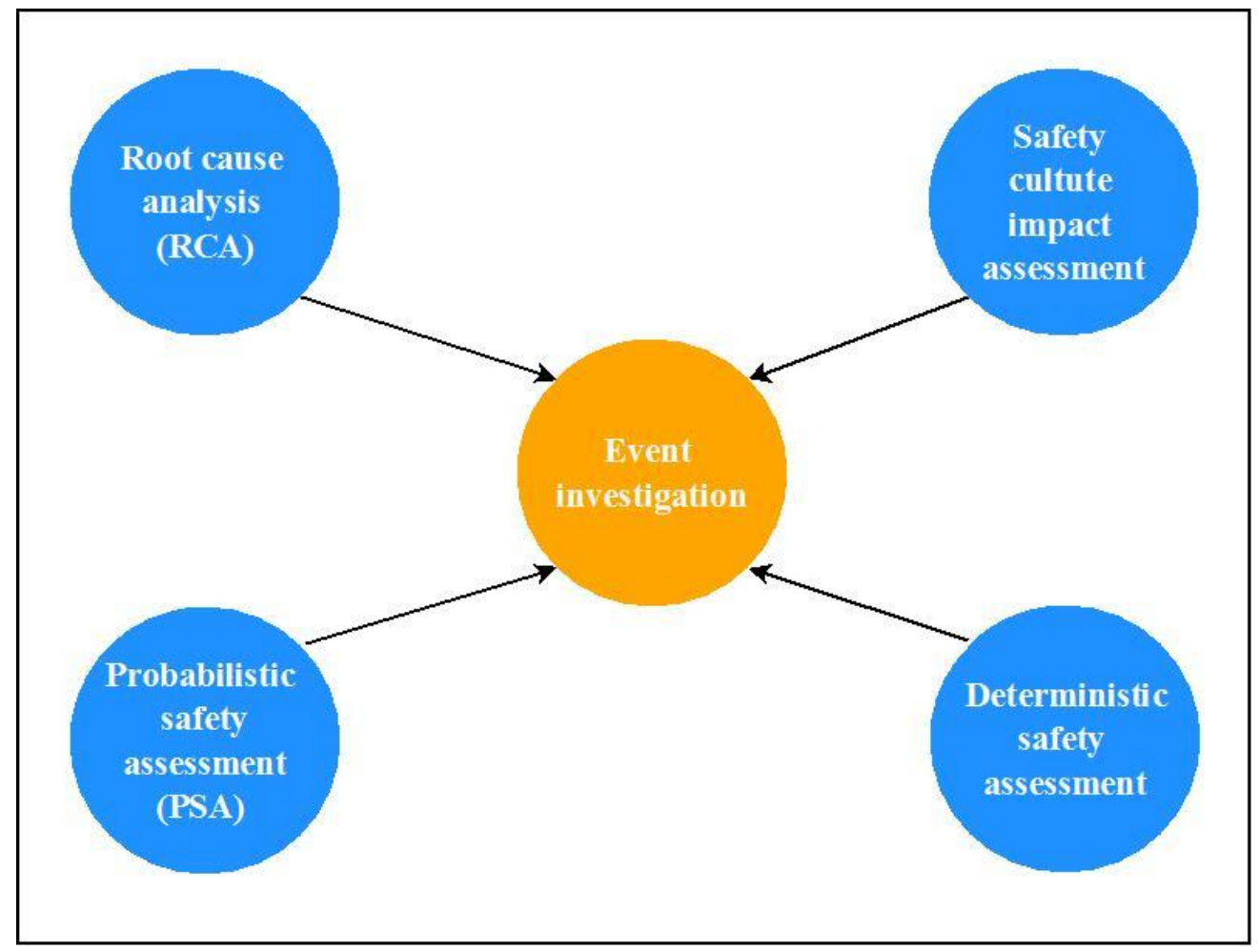

Figure 1 : Event investigation methodologies used in nuclear industry

For operating events, the most common methodology used during the investigations is RCA. The necessity to investigate in depth complex events demands by several tools that have been developed. Some of famous tools are: human performance enhancement system (HPES), management oversight and risk tree (MORT), assessment of safety significant event team (ASSET) [16].

\subsection{Event investigation methods}

HPES uses the graphical representation to investigate events combining cause and effects, based on direct causes, root causes, contributing causes and failed barriers [16]. Fig. 2 shows a hypothetical event and casual factor chart (ECFC) where the consequence is a reactor shutdown. 
The shutdown signal occurs when the condensate flow achieves a value below the minimum set point after the plant starting up. To demonstrate the application of the methodology, only a flow signal is tested for the value of $145 \mathrm{~kg} / \mathrm{s}$, but in real design there are redundant signals to increase the safety level. Considering a set of possible root causes was considered a deficiency during the specification of a flow transmitter. This deficiency considers that the transmitter was installed in an environment incompatible with its qualification.

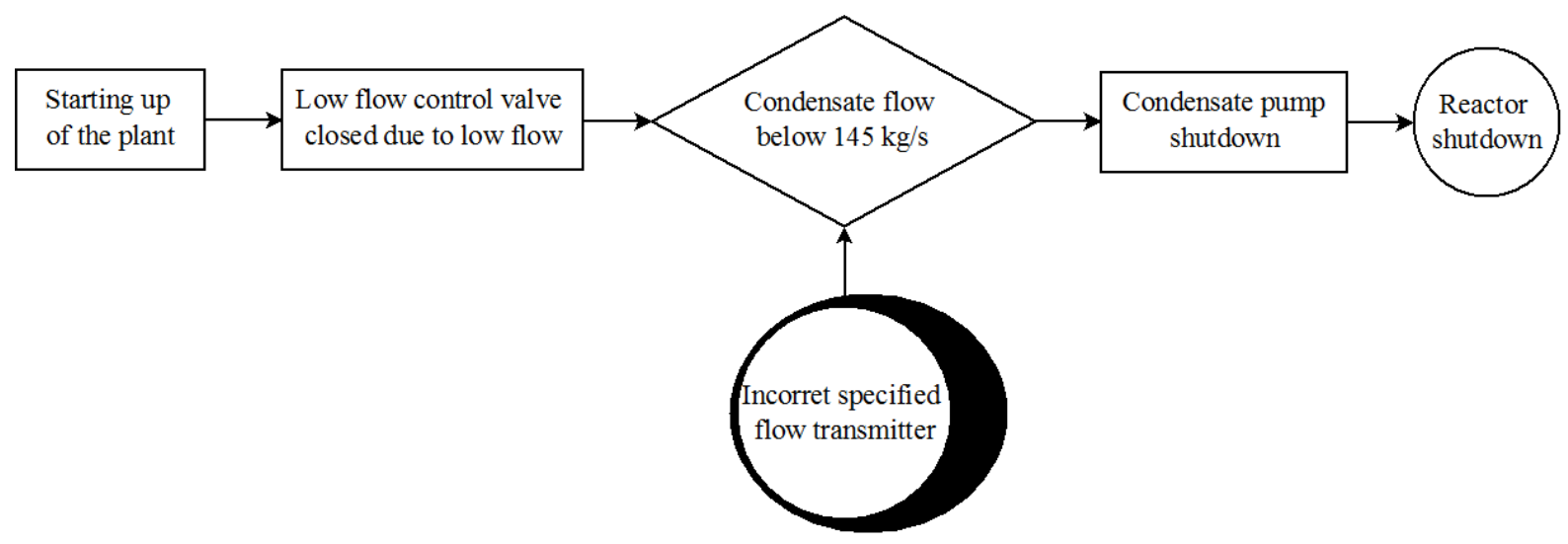

Figure 2: Example of ECFC for an event reactor shutdown

MORT uses a logic tree diagram to identify possible losses considering assumed risks, oversights and omissions, based on specific or management systems factors [16][19]. Fig. 3 shows a hypothetical example of MORT for an explosion in electrical generator building. This type of event has a probability to occur since the electrical generator has a hydrogen atmosphere. For this example, the explosion is associated to a leakage of hydrogen and the presence of an ignition source. A combination of organization culture related to smoking and deficiencies in maintenance can result in an accident. 


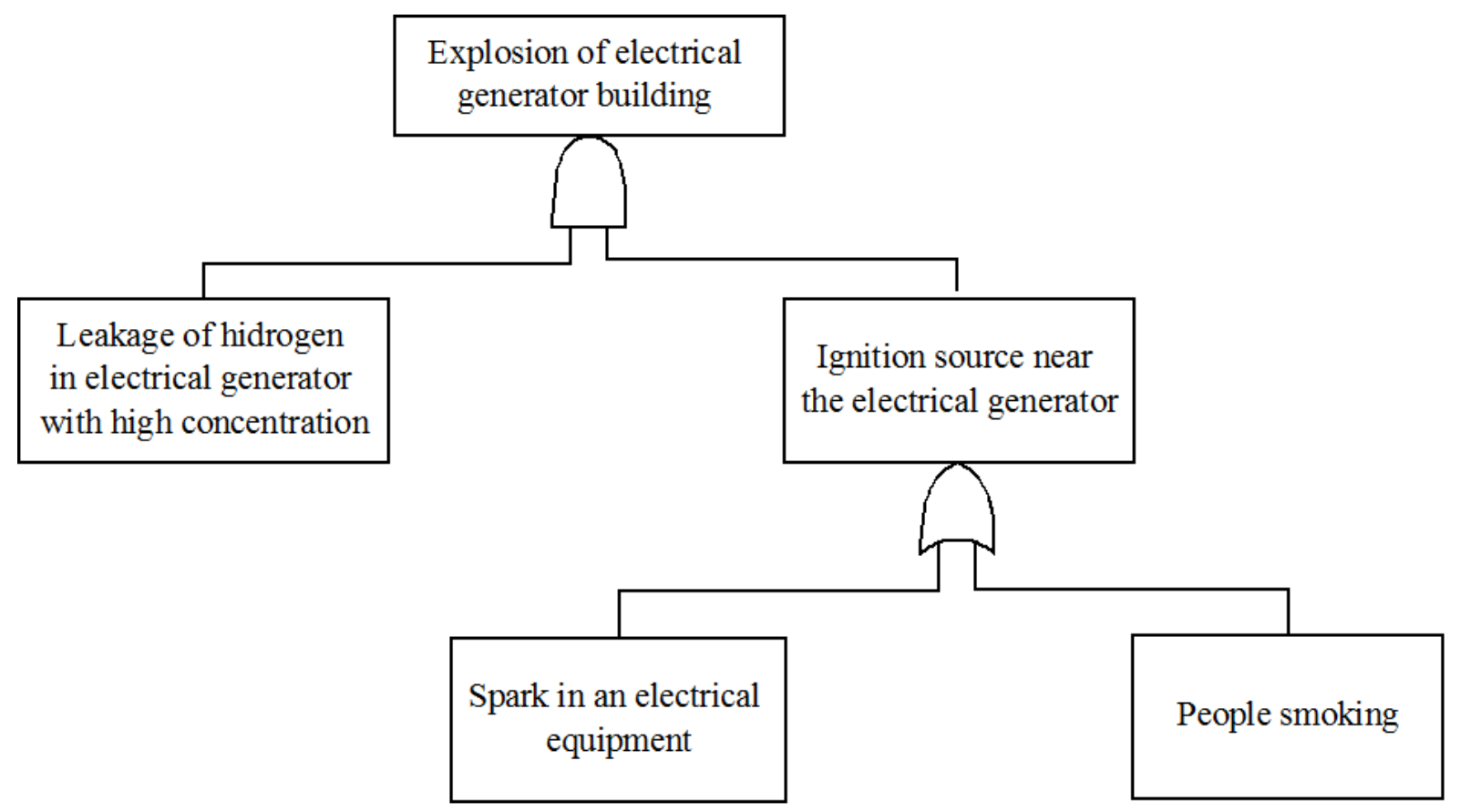

Figure 3 : Example of MORT for an explosion in electrical generator building

ASSET is an investigation methodology of high level and significance to analyze "managerial and organization issues". This methodology consists of three phases: investigation, to determine what happened; analysis the occurrences; and formulation of recommendations. Limitations of application of ASSET are related to differences on terminology and definition of root causes and the establishing of correctives actions only for high level and support [16].

\subsection{International Reporting System}

To support Operating Experience Feedback in nuclear industry, the NEA and IAEA developed a System that permits exchange of experience between different countries, called International Reporting System [20]. This system has information about most important events assessment in each country that is presented and discussed in an annual meeting. Each country has a local coordinator that includes events information and classified in accordance with IAEA guide. The local coordinator also has the function to permit and control the access of people on the system 
based on motivation for use the information registered. Each report is evaluated by a group of specialists before publishing. Because of this necessity, was developed an IRS Guideline that permits standardization event information considering different national reporting requirement and country maturity level. The coding is divided in nine groups [20]:

1) Reporting category;

2) Plant status prior to the event;

3) Failed/Affected systems;

4) Failed/Affected components;

5) Cause of the event;

6) Effects on operation;

7) Characteristics of the event/issue;

8) Nature of failure or error;

9) Recovery actions.

\section{RESULTS AND DISCUSSION}

This paper evaluates 408 events registered in IRS between 2014 and 2018. This evaluation highlights the most recurrent code for each group. In several cases an event can be more than one classification in a same group and, because this, the sum of records can be higher than the number of events. Presented graphics are considering the most relevant class related to a group. During the research were identified codes in reports that don't exist in the version of 2010 of IRS Guidelines.

Fig. 4 demonstrates that in a set of 26 options, the most frequent reporting category is related to "deficiencies in operation", corresponding for $18 \%$ of the occurrences analyzed. Another relevant category is related with "events of potential safety significance" that occurred in $12 \%$ of all occurrences. 


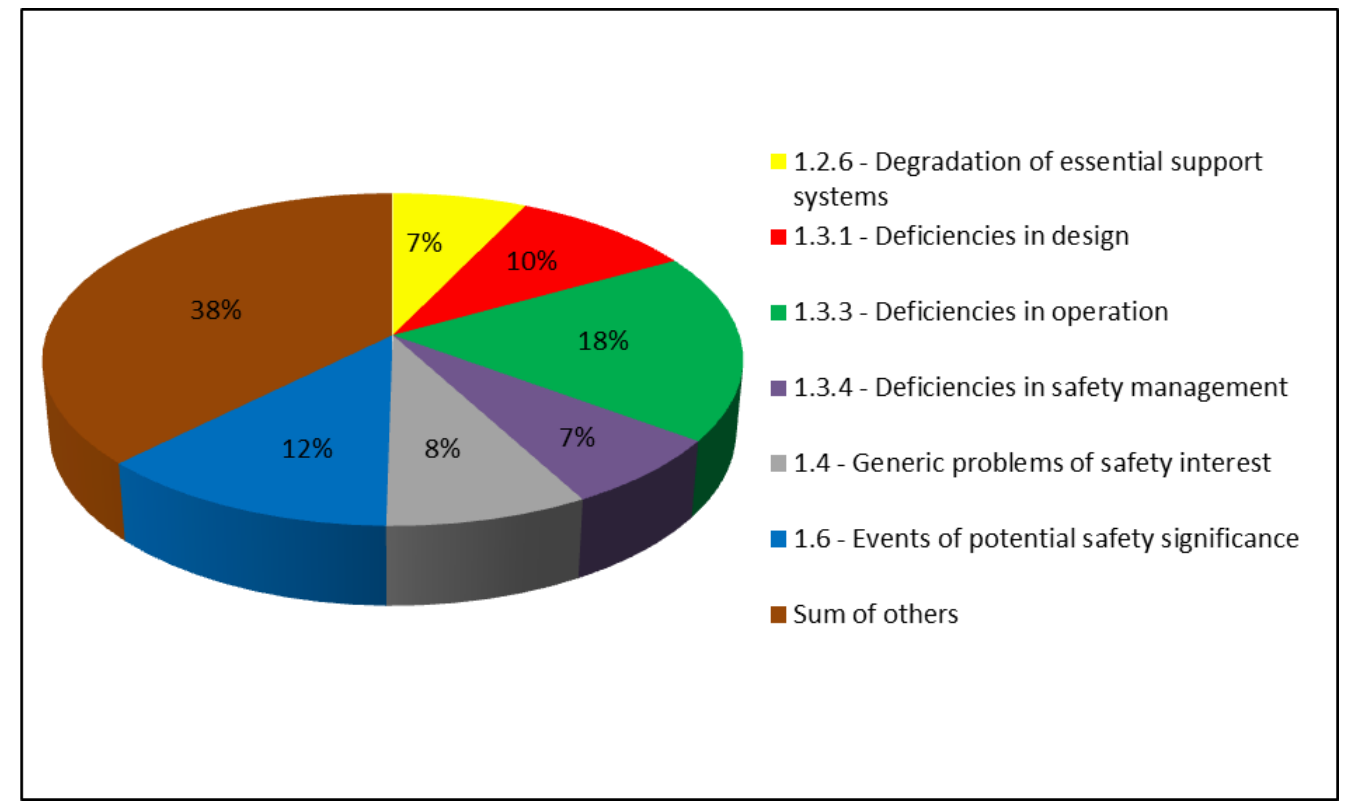

Figure 4: Reporting category

The most recurrent plant status founded in IRS database is "full allowable power" with $33 \%$ of occurrences in a set of 23 possibilities, as showed in Fig. 5. In 8\% of occurrences, plants were in testing or maintenance. Another recurrent classification was "On power", representing 8\% of the set of status.

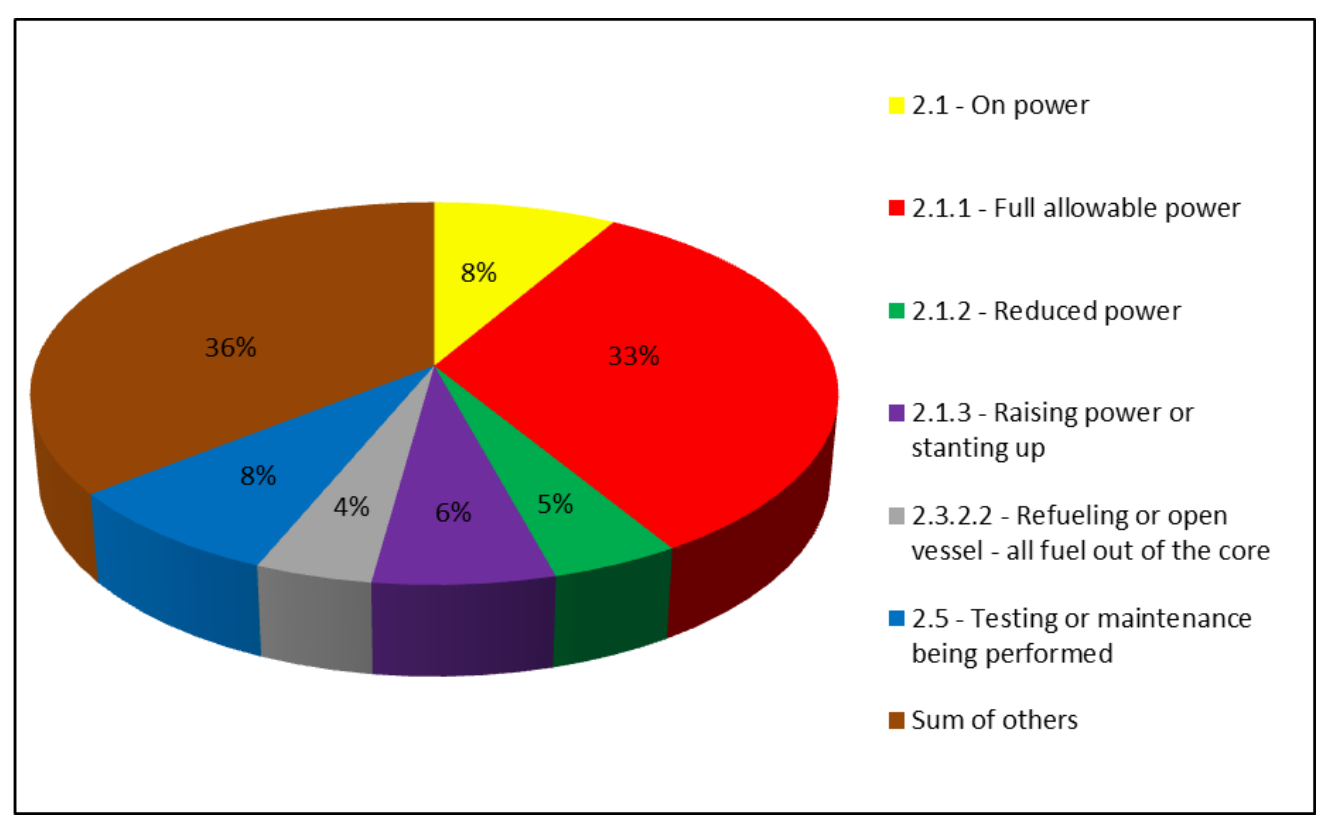

Figure 5: Plant status prior to the event 
In a set of 133 groups of system, the most cited code for events related to system was "emergency power generation and associated auxiliaries" with 7\%. The "primary coolant system" and "emergency core cooling" appeared with 4\% of the total events as shown in Fig. 6.

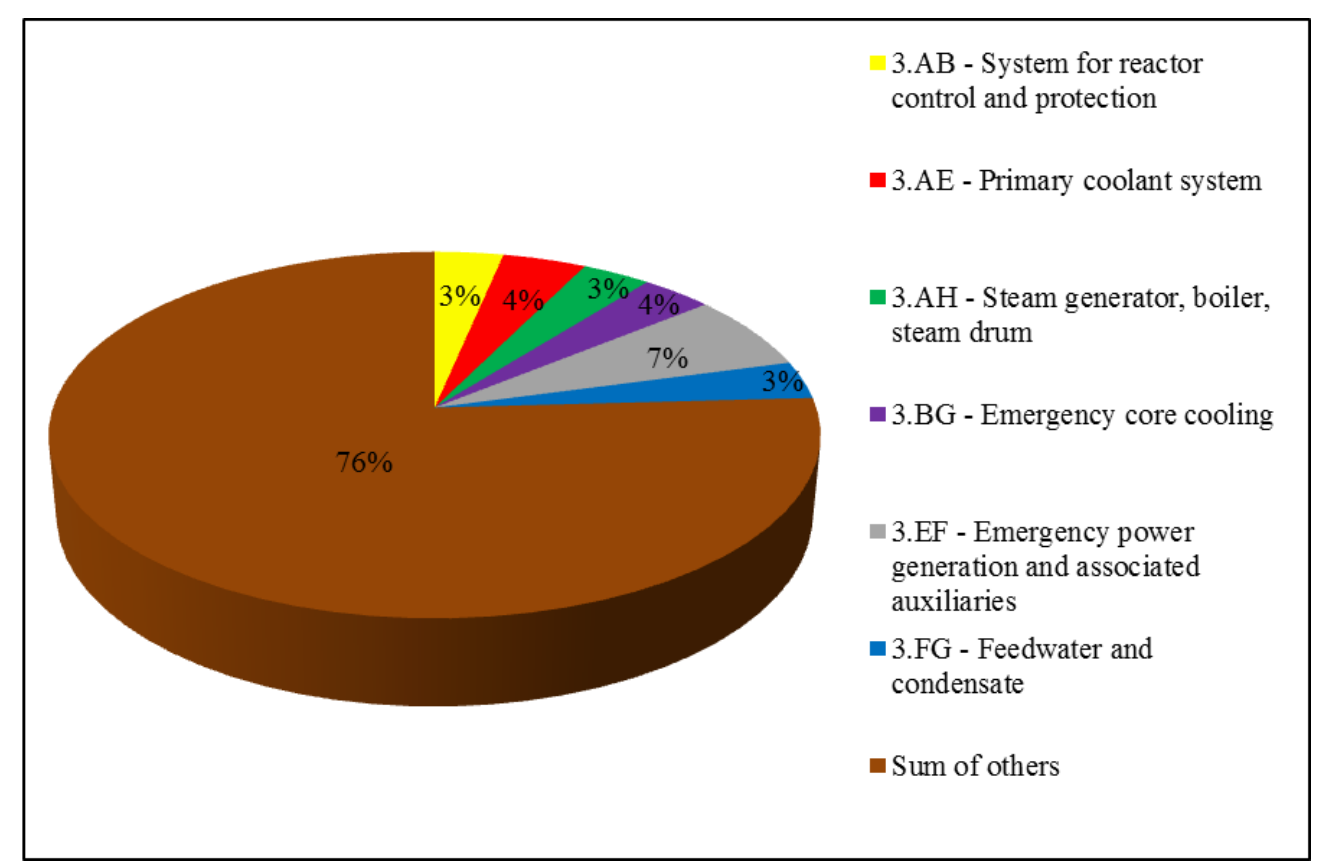

Figure 6: Failed/affected system

Considering a set of 50 groups of components that can be considered during the event investigation, in $14 \%$ of occurrences the most classified was "valves, valves operators, controllers, dampers and fire breakers, seals and packing", like showed in Fig. 7. The second group most cited was "tubes, pipes and ducts" in $8 \%$ of the possibilities. 


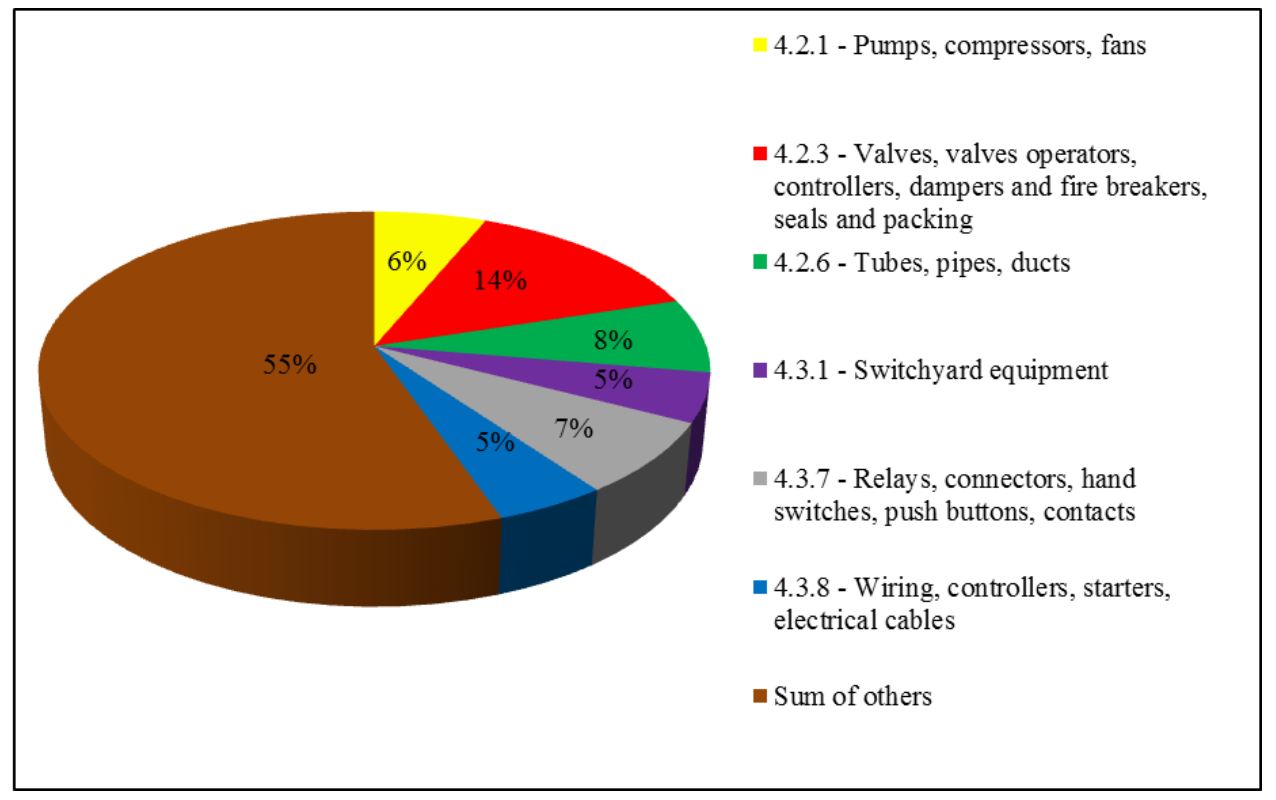

Figure 7: Failed/affected component

Fig. 8 illustrated that between 167 listed code for cause in IRS Guideline, the most cited was maintenance, in $4 \%$ of the occurrences. After that, with 3\% appears code for "Operations", "Procedure completeness/accuracy" and "Maintenance, testing or surveillance". In this group is possible to identify the possibility of mistakes related to maintenance that is cited in two codes.

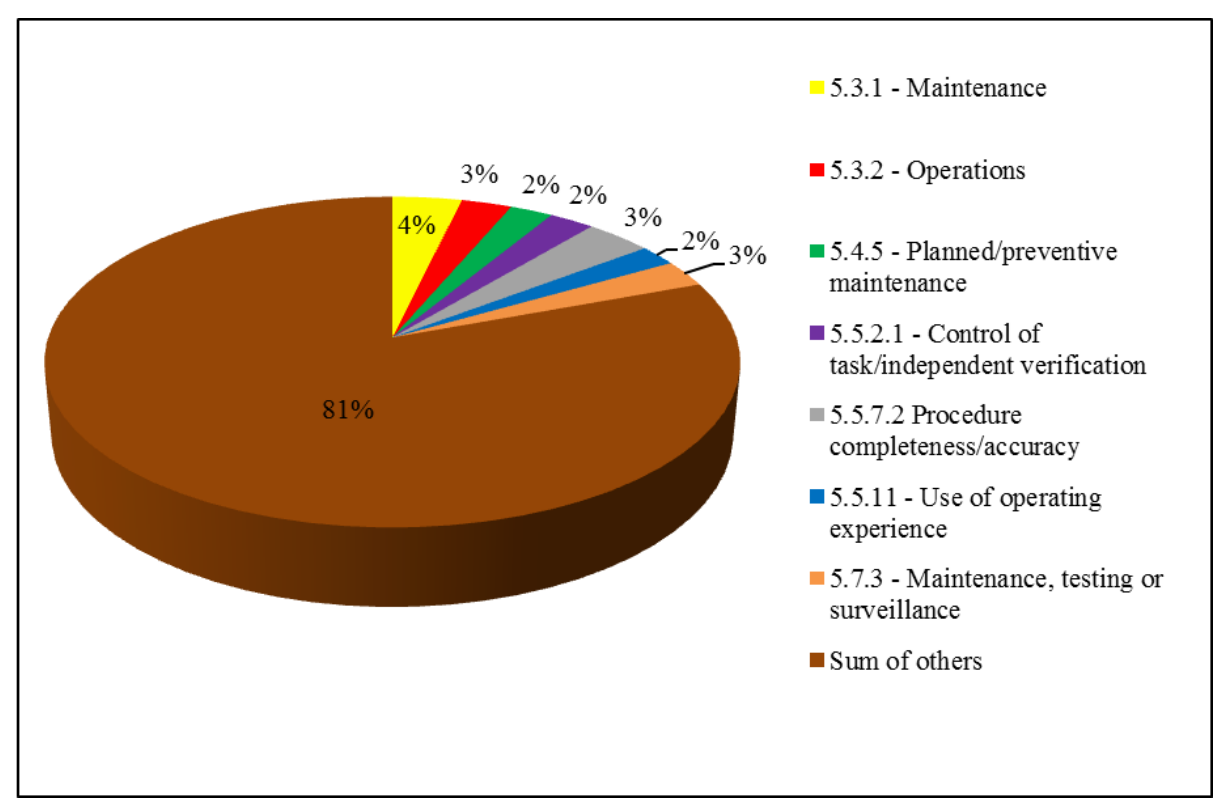

Figure 8 : Cause of the event 
In $37 \%$ of events investigated in this research no significant effect were registered. The most recurrent effect, in a set of 20 possibilities, was the reactor scram. Automatic scram, that occurs without operator' actions, occurred in $13 \%$ of the cases while in $10 \%$ occurs an "exceeding technical specification limits". Fig. 9 shows the most important effects in the operation for registered IRS events.

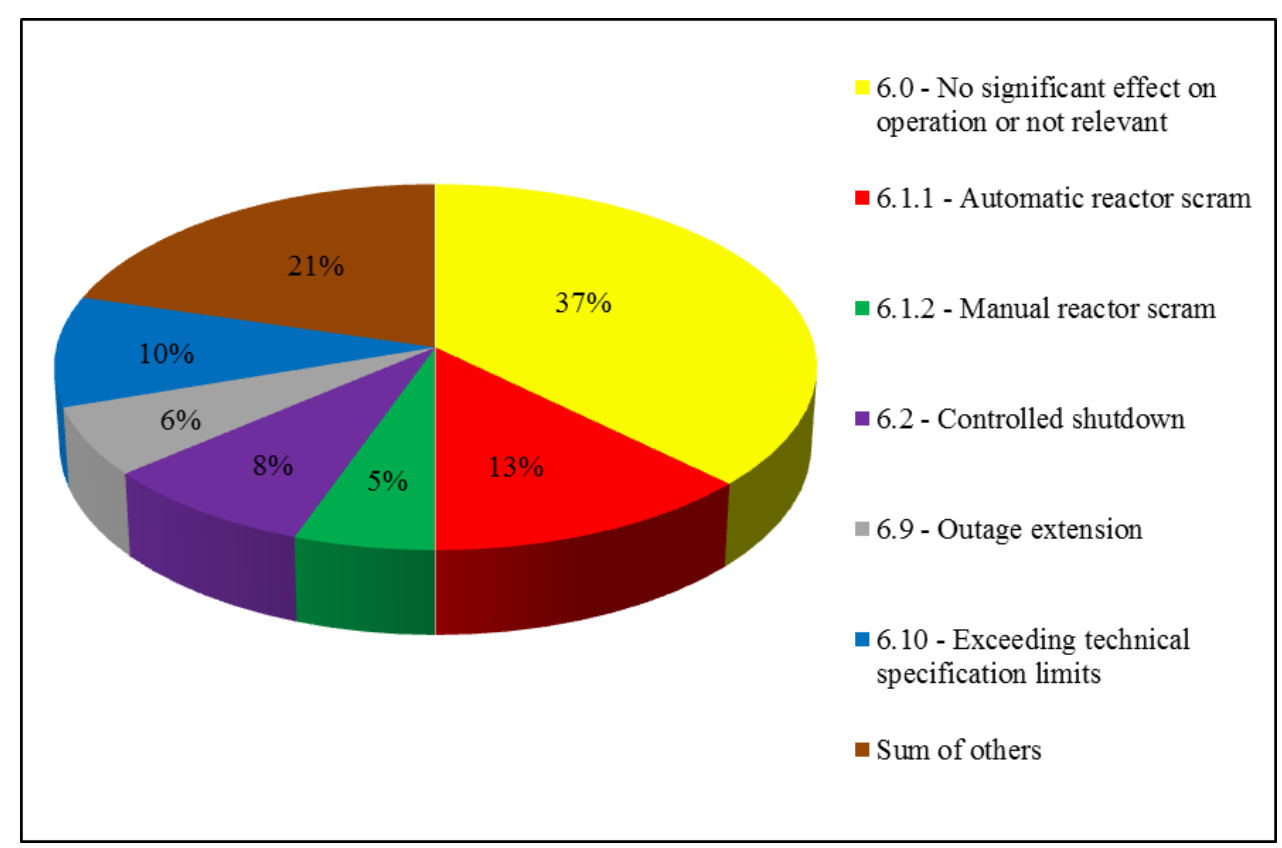

Figure 9: Effects on operation

Between 22 possible characteristics for events showed in Fig. 10, the most cite was "Other characteristics", however, the guide don't have clear information for this category. In $13 \%$ of the cases were observed a "significant degradation of safety function". With $7 \%$ of occurrence, were reported events related to "power transient", "physical hazards" and "discovery of major condition not previously considered or analyzed". 


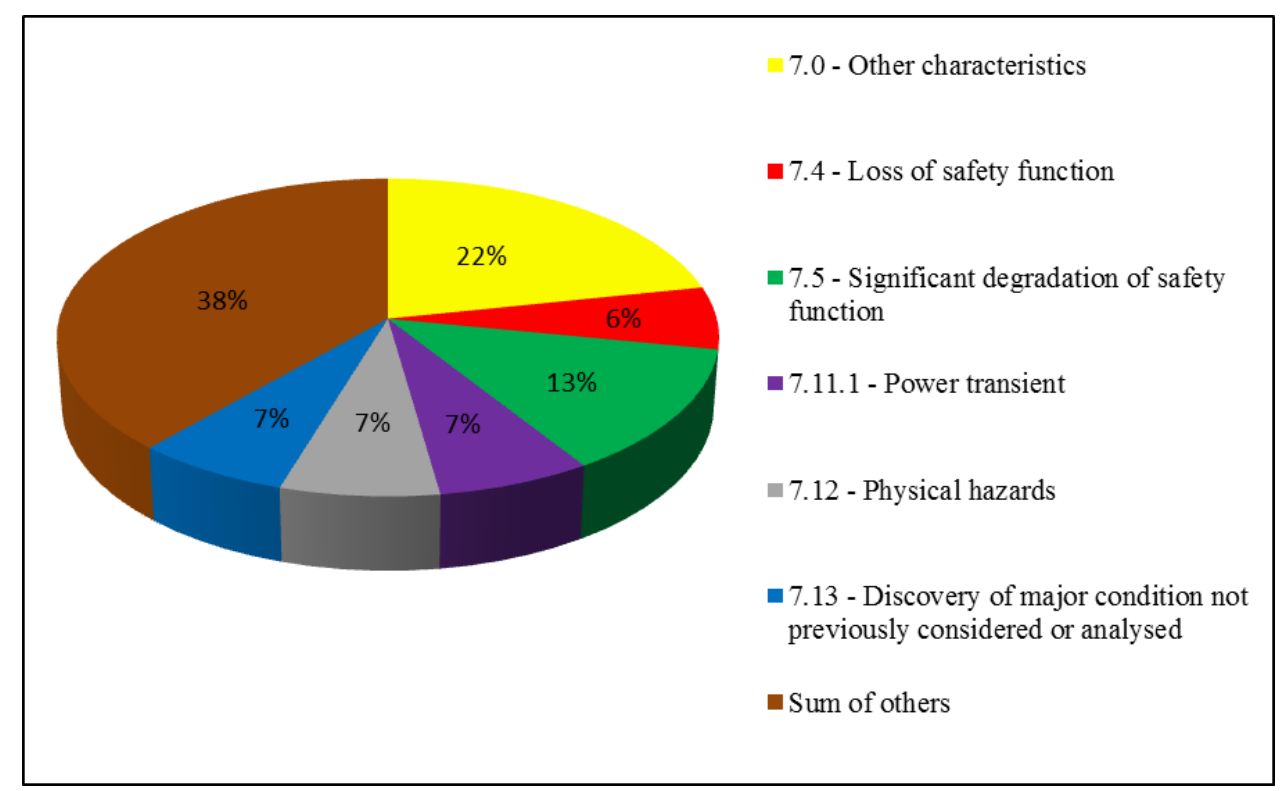

Figure 10: Characteristics of the event/issue

"Single failure or single error" corresponds to $30 \%$ of the observed events and it's showed in Fig. 11. Another relevant code was related to a Common cause failure (CCF) in $19 \%$ of the cases and has big importance considering that redundancy is a solution to increase the reliability in accordance with defense in depth principles [21].

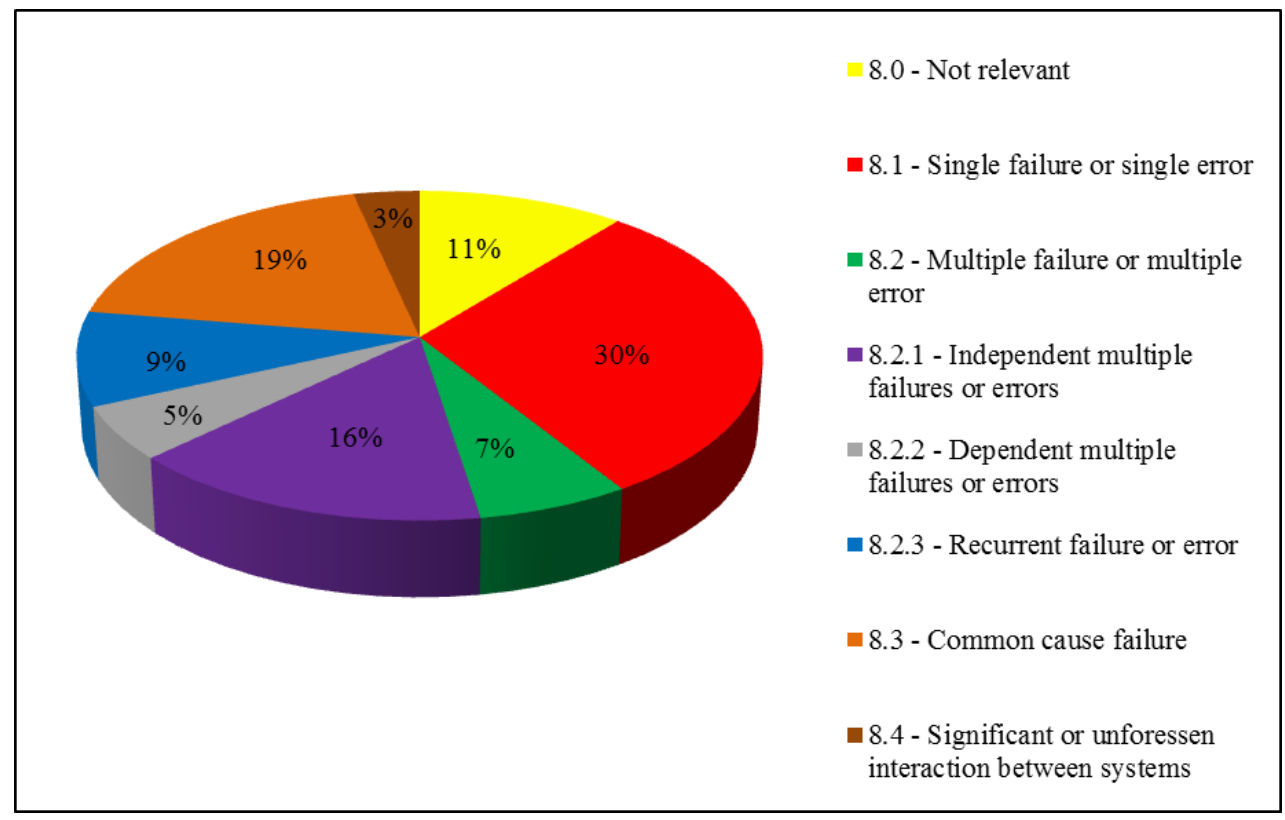

Figure 11: Nature of failure or error 
Fig. 12 demonstrated that in $61 \%$ of the events the recovery was done by foreseen human action, indicating the importance of training and qualification of plant staff. Other $19 \%$ of events were classified by human action. With $10 \%$ of occurrence were recovery by unforeseen human action and automatic plant action. The classification using these codes can promote a confusing since there isn't a boundary definition between normal and foreseen human action.

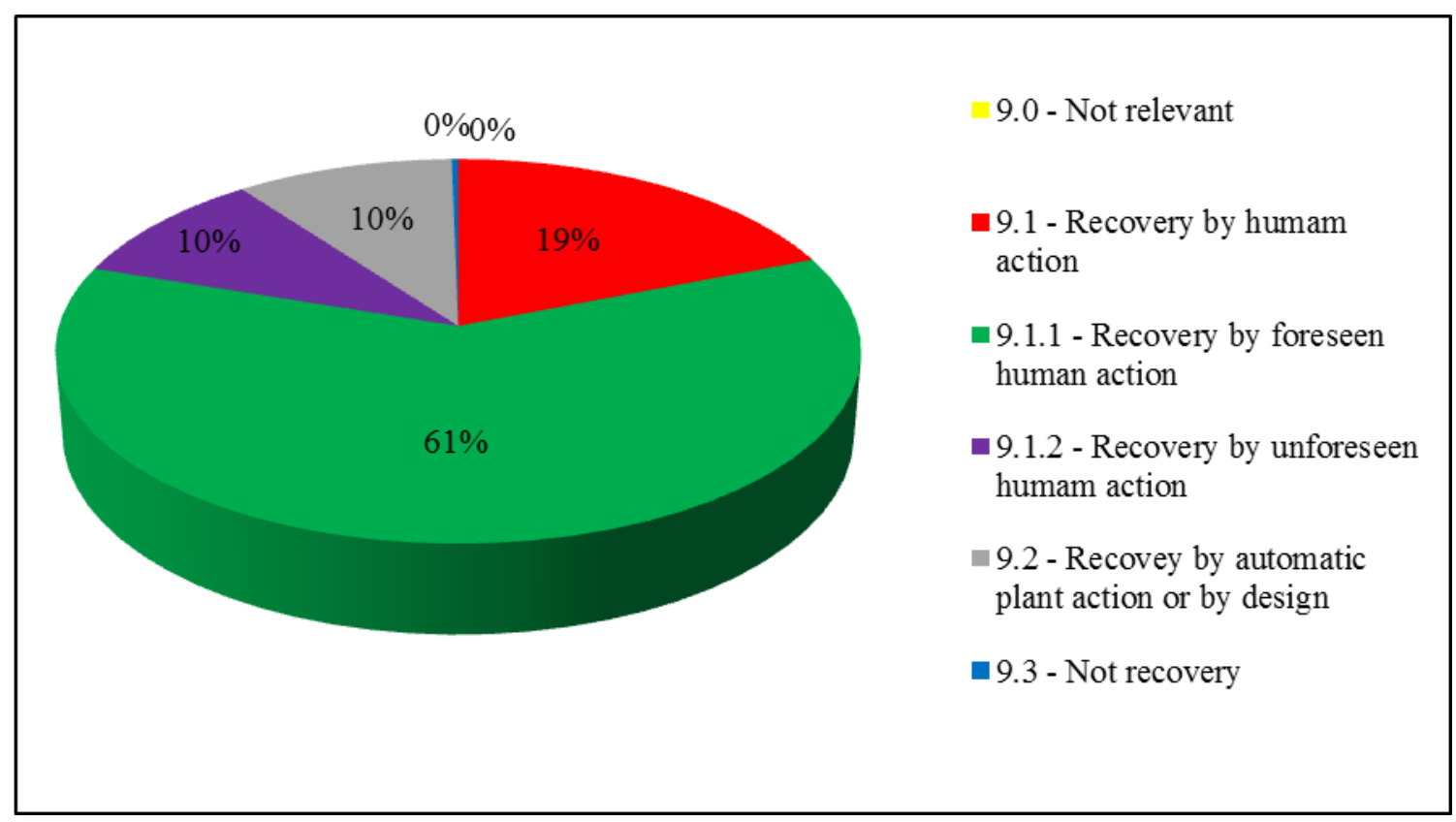

Figure 12: Recovery actions

\section{CONCLUSION}

This paper highlights the importance of operating experience feedback has for safety of nuclear installation. Another important conclusion is the good practice and tool in using of a good database to identify the most recurrent characteristics of events and take correct actions to reduce the occurrences of errors and limiting the consequences. Some results like recovery indicate the importance of qualification and training of the staff and organizational factors like good procedures, safety culture and human performance tools. The conclusion about cause of events demonstrated the importance of goods programs of maintenance to reduce equipment degradation or necessity of replacement. 
In this work was demonstrated the characteristics of IRS and the guide developed by IAEA. Also, it was identified points to improve like a revision of the guideline to include new codes. Another space for improvement, consist to define better some description for some codes.

\section{REFERENCES}

[1] NEA - Nuclear Energy Agency. Comparing Nuclear Accident Risks with Those from Other Energy Source, Paris, France, 2010.

[2] NASA - National Aeronautics and Space Administration. Lessons learned from Challenger, Washington D.C, USA, 1988.

[3] MEDEIROS, L. O. Abordagem da ergonomia para avaliação do treinamento simulado das equipes de resposta à emergência em lançamentos de veículos aeroespaciais. Dissertação de Mestrado. Natal: Universidade Federal do Rio Grande do Norte, 2015.

[4] MOREIRA, J. F. M., d'ALMEIDA, A. L. Indústria de petróleo e gás: acidentes relevantes no mundo. III Congresso Nacional de Engenharia de Petróleo, Gás Natural e Biocombustíveis, Salvador, Bahia, Brazil, 2018.

[5] LE COZE, J. C. BP Texas city accident: weak signal or sheer power?. Resilience Engineering Symposium, Antibes, Juan-les-Pins, France, pp. 185-194, 2008.

[6] ISIADINSO, C. BP Texas City refinery disaster - accident \& prevention report. Exeter, United Kingdom, 2015.

[7] MEJRI, M., de WOLF, D. Crisis management: lessons learnt from the BP Deepwater Horizon Spill Oil. Business Management and Strategy, v. 4, pp. 67-90, 2013.

[8] PEDRAZA, J. M. World major nuclear accidents and their negative impact in the environment, human health and public opinion. International Journal of Energy, Environment and Economics, v. 21, 2013.

[9] REMPE, J., FARMER, M., CORRADINI, M., OTT, L., GAUNTT, R., POWERS, D. Revisiting insights from Three Mile Island Unit 2 postaccident examinations and evaluations in view of the Fukushima Daiichi Accident. Nuclear Science and Engineering, v. 172, pp.223-248, 2012. 
[10] KORTOV, V., USTYANTSEV, Y. Chernobyl accident: Causes, consequences and problems of radiation measurements. Radiation Measurements, v. 55, pp. 12-16, 2013.

[11] MAURICIO, C. L. P. Analysis of the external doses received by workers involved in the mitigation of the Goiania radiological accident. Brazilian Journal of Radiation Sciences, v. 6, 2018.

[12] PYY, P., ROSS, D. F. Recurring events: a nuclear safety concern. NEA News, 21.2, 2003.

[13] IAEA - International Atomic Energy Agency. Best practices in the utilization and dissemination of Operating Experience at Nuclear Power Plants, Vienna, Austria, 2008.

[14] CNEN - Comissão Nacional de Energia Nuclear. Norma NN 1.14 - Relatórios de operação de usinas nucleoelétricas, 2002.

[15] IAEA - International Atomic Energy Agency. Safety of Nuclear Power Plants: Commissioning and Operation, Specific Safety Requirements (SSR-2/2), Vienna, Austria (2016).

[16] ZIEDELIS, S., NOEL, M. Comparative Analysis of Nuclear Event Investigation Methods, Tools and Techniques - Interim Technical Report EUR 24757 EN, European Commission Joint Research Centre - Institute for Energy, Luxemburg, Luxemburg, 2011.

[17] IAEA - International Atomic Energy Agency, Development and application of level 1 probabilistic safety assessment for Nuclear Power Plants, Specific Safety Guide (SSG-3), Vienna, Austria, 2010.

[18] IAEA - International Atomic Energy Agency, Deterministic Safety Analysis for Nuclear Power Plants, Specific Safety Guide (SSG-2), Vienna, Austria (2009).

[19] MOHAMADFAM, I., SOLEIMANI, E., GHASEMI, F., ZAMANPARVAR, A. Comparison of Management Oversight and Risk Tree and Tripod-Beta in Excavation Accident Analysis. Health Science, v. 7, pp. 1-5, 2015.

[20] IAEA - International Atomic Energy Agency. IRS Guidelines. Vienna, Austria, 2010.

[21] ERINC, J., SEAMAN, S. BAISCH, J. Common Cause Failure (CCF): A path to quantitative success. NPIC\&HMIT, San Francisco, CA, pp. 246-255, 2017. 